\title{
IN-SITU PRECISE ANGLE MONITORING ON SYNCHROTRON RADIATION MONOCHROMATOR BY USE OF PENCIL BEAM INTERFEROMETER*
}

Shinan Qian, Peter Takacs, Qinli Dong, and Steve Hulbert

Brookhaven National Laboratory

Upton, NY 11973

September, 2003

"Work supported by the U.S. Department of Energy: Contract No. DE-AC02-98CH10886. 


\title{
In-situ Precise Angle Monitoring on Synchrotron Radiation Monochromator by Use of Pencil Beam Interferometer
}

\author{
Shinan Qian ${ }^{*}$, Peter Takacs ${ }^{*}$, Qinli Dong ${ }^{\dagger}$, Steve Hulbert ${ }^{\dagger}$ \\ "Instrumentation Division \\ ${ }^{\dagger}$ National Synchrotron Light Source \\ Brookhaven National Laboratory, Upton, NY 11973 USA
}

\begin{abstract}
Monochromator is a very important and precise instrument used in beam lines at synchrotron radiation facilities. We need to know if there is actual thermal distortion on gratings resulting in the degradation of the monochromator resolution. We need to know the characteristics of the grating rotation. It is possible to make a simple but precise in-situ distortion monitoring and rotation angle test of the grating by use of a precise pencil beam angle monitor: We have made preliminary measurements on a monochrometer grating of an undulator beam line X1B at Brookhaven National Laboratory. We monitored a small amount of angle variation on the grating. We detected $1.7 \mu \mathrm{rad}$ backlash (P. V) of the grating controlling system.
\end{abstract}

\section{INTRODUCTION}

In-situ distortion testing is helpful in improving the quality of synchrotron radiation beam lines. The first on-linedistortion measurement was made on the first mirror of the beam line at ELETTRA in Italy in 1995, which was exposed to a total power of about $200 \mathrm{~W}[1,2]$. Another in-situ test was performed on the third mirror of a beam line at the APS at Argonne National Laboratory [3], which was subject to a total power of about $100 \mathrm{~W}$. In-situ measurement of distortion of a diffraction grating has not yet been accomplished.

The monochromator is a very important and precise instrument used in beam lines. Thermal distortion of the grating surface figure will result in degradation of the monochromator resolution. However, a grating is generally located downstream in the beam line as the third or fourth optical component, and is usually subject to a smaller heat. load than the first or second reflecting elements.

The rationale for testing a grating is to learn if thermal distortion effects are evident on the grating figure even: though the total power is very small, and to evaluate an easy and quick technique for distortion measurement using stationary probe beams without the need for a scanning stage. In order to test small angle variations, a sensitive. precision angle monitoring (PAM) system is used [4].

The gratings under test are installed in a monochromator on beam line X1B at the National Synchrotron Light Source (NSLS) at Brookhaven National Laboratory. The test result shows that a small distortion can be seen on the grating although it is of small enough magnitude that it has negligible effect on the system resolution at the present time. This experiment also shows that the grating control system has small backlash of about $1.7 \mu \mathrm{rad}$. The in-situ monitoring method provides a precision diagnostic for calibrating the grating rotation angle mechanism under actual operating conditions.

\section{THE GRATING UNDER TEST}

The grating under test (GUT) is used on X1B beam line (Fig. 1), the soft Xray spectroscopy undulator beam line,

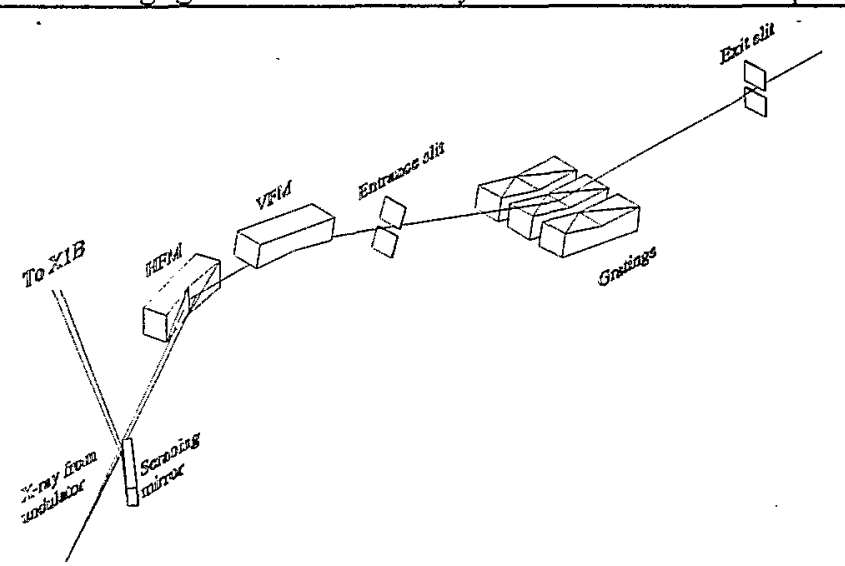

Figure 1: The grating under test on beam line X1B of NSLS at BNL 
at NSLS [5]. This beam line accepts the $x$-ray flux from an undulator with a maximum power of about $400 \mathrm{~W}$. The first optical element of X1B is a scraping mirror that divides the beam into two parts by a wavefront-splitting method. One part of about $35 \%$ flux is deflected by the scraping mirror to the X1A beam line; the remaining $65 \%$ flux goes directly to $\mathrm{X} 1 \mathrm{~B}$. Immediately downstream are a horizontal focus mirror (HFM) and a vertical focus mirror (VFM) and the entrance slit in front of grating. Both mirrors are water cooled from the side in order to minimize distortions. The grating is the fourth optical component downstream from the source and accepts a very small power of about $20 \mathrm{~W}$. The grating is spherical fused silica ( $R=57.3$ meters) in dimensions of $170 \times 50 \times 30 \mathrm{~mm}$, water cooled along the sides. The full deflection angle of the grating is 6.6 degrees.

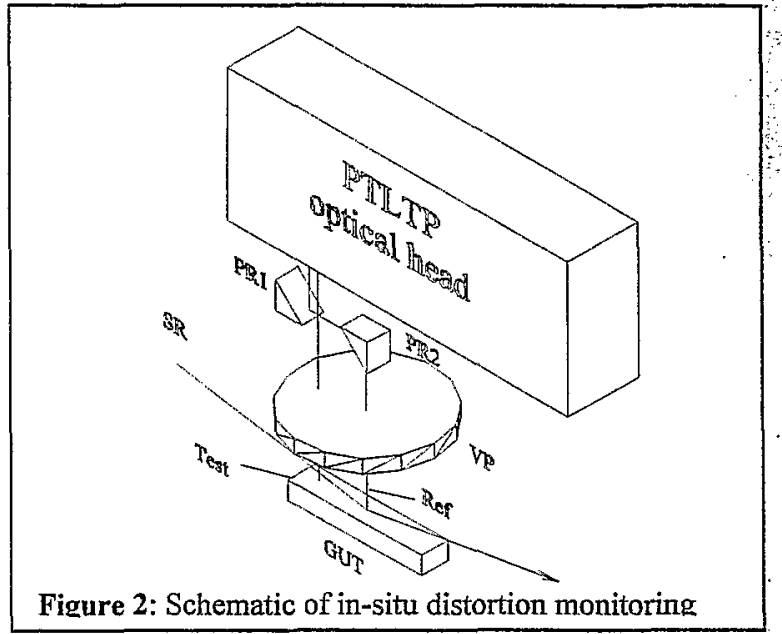

\section{SETUP OF THE TEST EQUIPMENT}

As described above, for simplicity and ease of monitoring, we only used stationary probe beams without scanning them. This enables us to measure angle variations at specific points in order to estimate distortion of the entire surface. We used an optical head of the Portable Long Trace Profiler (PTLTP) as a precise angle monitoring (PAM) system, which provides two probe beams to the grating under test (GUT) [5].

The schematic of the in-situ distortion monitoring system is shown on Fig. 2. The optical head of the PTLTP is easily fixed to a 130 $\mathrm{mm}$ diameter flange of the vacuum view port (VP), and is aligned to send two parallel sets of probe beams down to the GUT. One set beams go through VP directly to a point located as far as possible from the center of the GUT in order to monitor larger angle variation. The distance between the probe point and the center of the GUT is about $60 \mathrm{~mm}$. Another set beams are reflected by two prisms (PR1 and PR2) and are sent to the center of the GUT as a reference

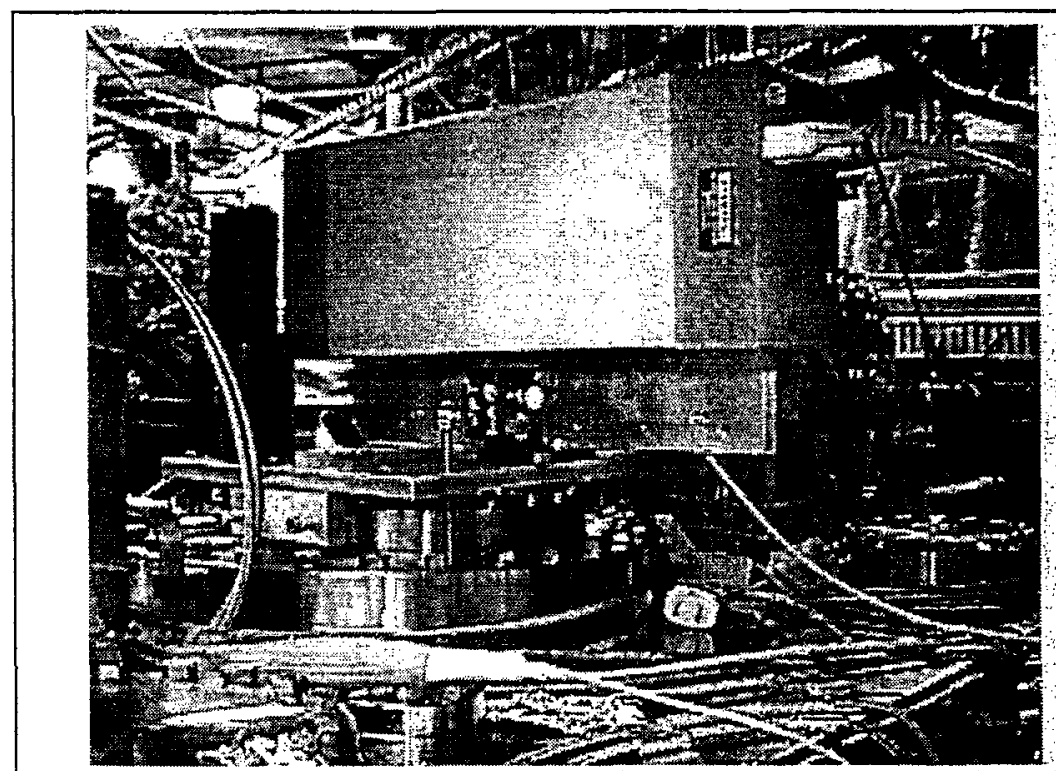

Figure 3: Photo of the in-situ distortion monitoring equipment set up at beam line $X 1 B$ at NSLS

beam for checking if the grating undergoes a rigid body rotation during the test. Theoretically the center point of the grating will have no angle variation if there is a symmetric thermal distortion. The beams are then reflected back to: the optical head of the PTLTP and are detected by a CCD camera to determine the angle variation. Fig. 3 is the photo of setup on beam line X1B. In this test everything is fixed to the top chamber flange.

\section{SOME TEST RESULTS}

\section{Thermal distortion test}


The monitoring was done by opening and closing the beam line shutter and switching the cooling water on and off alternately for a larger distortion while taking stability scan. If the GUT is distorted by bending due to the thermal condition variation, the reflected beam will change direction. Fig. 4 shows the grating distortion test result. The large slope steps are clear evidence for distortion of the GUT. During the distortion test the storage ring current was $286 \mathrm{~mA}$ with the undulator gap at 36 $\mathrm{mm}$. The total power on the grating was estimated to be $20 \mathrm{~W}$.

There was no $\mathrm{X}$-ray beam on the GUT for a long time until point 45 , so the grating is cold. At points 45 and 145 the beam was turned on combined with water-cooling off, but at points of about 95 and 195 the beam was turned off with water-cooling on. Because the sudden heat load hits the cold grating at point 45 , there: is a bigger slope step by thermal distortion. The step heights subsequently decrease as the grating slowly warms up: The beam shutter of X1B was operated in a manual way through a lead screw that requires 5-10 seconds to open: orclose. There are strange slope jumps at points where the SR beam is switched on or off. These are. caused by the: force of hand-cranking the lead screw, which distorts the vacuum pipe and monochromator chamber. The noticeable slope distortion of $1-1.5 \mu \mathrm{rad}$ over the $60 \mathrm{~mm}$ separation distance is related to the $0.14 \%$ change in radius: of: curvature if we assumed a spherical distortion. If the grating is operated in a normal experiment.condition with the $\mathrm{X}$-ray beam and the water-cooling are always on, the distortion will be much less. So the impact of thermal distortion for X1B is negligible. The displacement of the reflected beam on the VP is very small, so the VP will not: create the test error. This monitoring test was set up and done very quickly in order to estimate the feasibility for: future diagnostics. Distortion measurements with a scanning profiler should follow if precision measurements. are deemed necessary $[1-3,7]$.

\section{Mechanical backlash test}

We tested grating rotation backlash of X1B beam line by making a stability scan with the PAM by rotating the grating forward and backward with a decreasing step value until the PAM can no longer see an angle variation step on the tested slope. Fig. 5 shows the test results. On curve (c), no rotation step can be seen with a backlash of about $1.7 \mu \mathrm{rad}$ ( $0.34 \mathrm{arc}$ second), which indicates that the mechanism is working well. The actual rotation angles of curves (a) and (b) are less than the theoretical driving angle according to the test. This is probably also cause by backlash.

\section{Calibration of grating rotation}

This is an introduction of a proposed way to calibrate rotation angle of the grating by use of the PAM. Possible test angles are restricted by the limited working range of the PAM. The calibration procedure is as follows: during: $\mathrm{a}$ stability scan of the PAM, the grating is rotated step by step, then a step-like slope curve (Fig. 6) is obtained.. By comparing the desired grating angle controlled by the program with the rotation angle measured by the PAM, the error can be calculated. The desired rotation steps are written on the curve of Fig. 6 . In this measuring process the

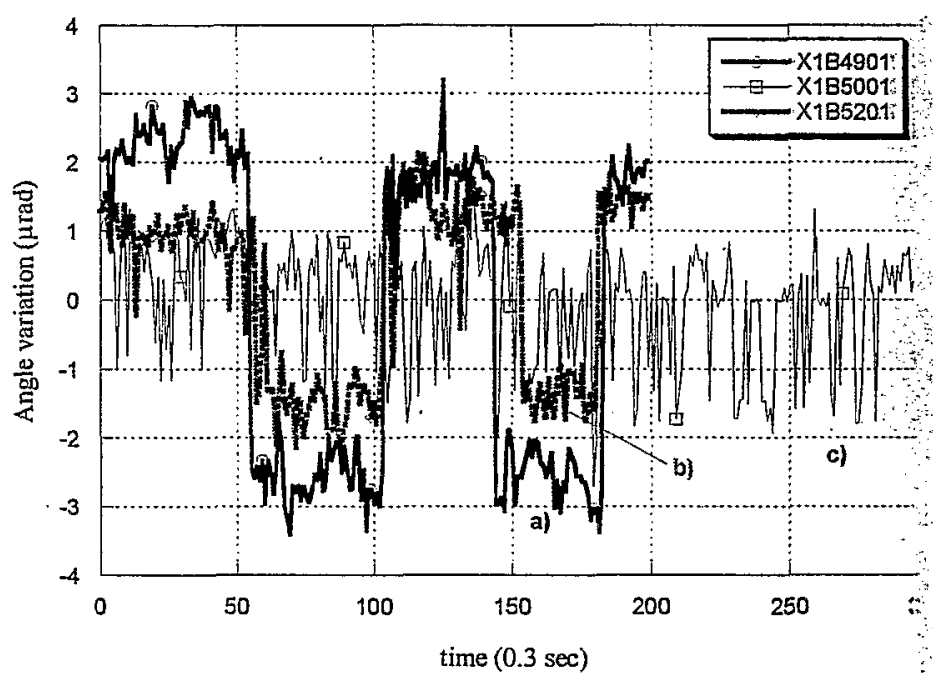

Figure 5: Grating backlash test by use of the PAM 
reflected beam scans over the VP over a larger distance, so a high quality VP is necessary, otherwise the VP will contribute an error signal. We have not done a precise test yet. Fig. 6 is an example of a test over a small angle range.

Other possible monitoring applications on synchrotron radiation beam lines:

1. Repeatability test of mechanics movement

2. Effectiveness test of water-cooling system by checking the distortion

3. Long turn stability test of mechanism

4. Precision orientation alignment of different changeable gratings

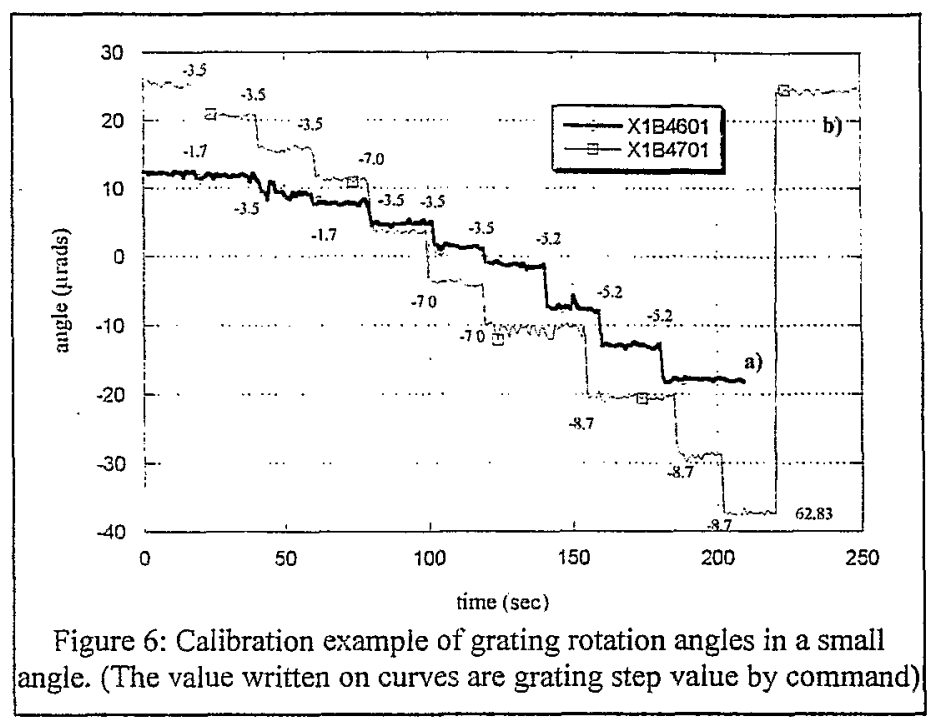

\section{DISCUSSION}

There are many advantages to monitoring real thermal distortion and angle variations in an easy and quick way by use of precise angle monitoring system for synchrotron radiation beam lines.

1. Convenient: easy to setup a test in a few days with the PAM or portable LTP

2. Simple: use one or two pencil beam(s) to monitor the local angle displacement to estimate the distortion quickly 3. Versatile: suitable for different kind of surfaces like flat, sphere, aspheric, small or large radius of curvature comparing to that restricted to plane surface by use of autocollimator.

4. Precise: it is easy to find the angle variation automatically as less as 1 urad, equal to 0.2 arc second.

5. Easier: easy to align because it uses a strong laser pencil beam.

For a convenient setup and reliable measurements, we are making a mini PAM for the future monitoring

\section{ACKNOWLEDGMENTS}

This research was sponsored by U. S. Department of Energy under contract No. DE-AC02-98CH10886.

\section{REFERENCES}

1. S. N. Qian, W. Jark, G. Sostero, A. Gambitta. F. Mazzolini, and A. Savoia, "Precise measuring method for detecting the in situ distortion profile of a high-heat-load mirror for synchrotron radiation by use of a pentaprism long trace profiler," Applied Optics, 36 (16), 3769-3775 (June 1997)

2. S. N. Qian, W. Jark, G. Sostero, A. Gambitta, F. Mazzolini, M. Tudor, A. Allemandi, A. Abrami, L. Batistello R. Sergo and A. Savoia, "Penta-prism LTP detects first in-situ distortion profile", Synchrotron radiation news, 9 (3), $42-44$ (1996)

3. P. Z. Takacs and S. N. Qian, K. J. Randall and W. B. Yun, H. Z. Li, "Mirror distortion measurements with an In-Situ LTP", SPIE, (1998)

4. S. N. Qian and P. Z. Takacs, "Precise angle monitor based on the concept of pencil-beam interferometry", Proc. SPIE, 4101, 263-272 (2000)

5. W. Eberhardt, K. J. Randall. J. Feldhaus, A. M. Bradshaw, R. F. Garrett and M. L. Knotek, "The Soft X-ray spectroscopy undulator beamline at NSLS X1", Physica Scripta 41

6. S. N. Qian and P. Z. Takacs, "Portable long trace profiler: concept and solution", Review of Science Instruments, Vol.72, No.8, 3198-3204, (2001)

7. S. N. Qian, W. Jark, P. Z. Takacs, K. J. Randall and W. B. Yun, "In situ surface profiler for high heat load mirror measurement", Opt. Eng., 34 (2), 396-402 (1995). 